\title{
Plane waves in a thermally conducting viscous liquid
}

\author{
BALJEET SINGH \\ Department of Mathematics, Government College, Sector 11, \\ Chandigarh 160 011, India \\ e-mail: baljeet_gill@hotmail.com
}

MS received 29 April 2002; revised 17 July 2003

\begin{abstract}
The aim of this paper is to investigate plane waves in a thermally conducting viscous liquid half-space with thermal relaxation times. There exist three basic waves, namely; thermal wave, longitudinal wave and transverse wave in a thermally conducting viscous liquid half-space. Reflection of plane waves from the free surface of a thermally conducting viscous liquid half-space is studied. The results are obtained in terms of amplitude ratios and are compared with those without viscosity and thermal disturbances.
\end{abstract}

Keywords. Thermally conducting; viscous liquid; plane waves; reflection; amplitude ratios; thermal relaxation times.

\section{Introduction}

The study of stress waves is of central interest to the engineers, geophysicists and seismologists due to its varied applications. The extensive literature on the subject is reviewed in the books of Ewing et al (1957), Bullen (1963), Bath (1968), Miklowitz (1966), Achenbach (1973), Kalski (1963) and by many other authors. The problems of wave and vibration become more interesting in the field of seismology, when we study the problems with the additional parameters (e.g. viscosity, thermal disturbance, porosity, microrotation, anisotropy, etc.).

Chadwick \& Sneddon (1958) and Lockett (1958) studied the propagation of thermoelastic plane waves. Knot (1899) derived the general equations for reflection and refraction at plane boundary. In classical dynamical-coupled theory of thermoelasticity, thermal and mechanical waves propagate with an infinite velocity, which is not physically admissible. To overcome this contradiction, the coupled theory of thermoelasticity has been extended by including the thermal relaxation time in constitutive relations by Lord \& Shulman (1967) and Green \& Lindsay (1972). Some problems on reflection in thermoelastic solid have been discussed by Deresiewicz (1960), Sinha \& Sinha (1974) and Singh (2000).

The present paper deals with the plane wave propagation in a thermally conducting viscous liquid half-space with thermal relaxation times. The reflection of the plane waves from the free surface of a thermally conducting viscous liquid half-space is discussed. 


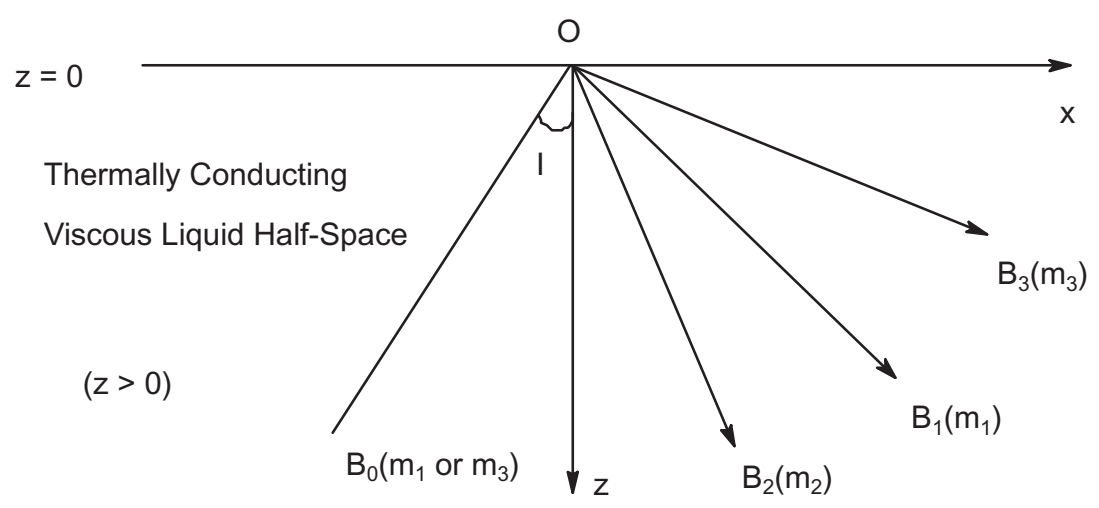

Figure 1. The geometry of the problem showing incident and reflected waves.

\section{Formulation of the problem}

We introduce rectangular Cartesian co-ordinates $(x, y, z)$ and place the origin at the free surface of a thermally conducting viscous liquid half-space as shown in figure 1 . The positive $z$-axis is taken into the half-space. Heat sources, external force loading and body forces are assumed to be absent. The constitutive equation and the equation of motion for a thermally conducting viscous liquid are (Lord \& Shulman 1967; Green \& Lindsay 1972)

$$
\begin{aligned}
& t_{k l}=\left(K-\frac{2}{3} \eta \frac{\partial}{\partial t}\right) u_{i, i} \delta_{k l}+2 \eta \frac{\partial}{\partial t} e_{k l}-\beta\left(T+\tau_{1} \dot{T}\right) \delta_{k l}, \\
& \left(K+\frac{4}{3} \eta \frac{\partial}{\partial t}\right) \nabla(\nabla \cdot \mathbf{u})-\eta \frac{\partial}{\partial t} \nabla \times(\nabla \times \mathbf{u})-\beta \nabla\left(T+\tau_{1} \dot{T}\right)=\rho \ddot{\mathbf{u}}, \\
& \rho C^{*}\left(\dot{T}+\tau_{o} \ddot{T}\right)+\beta T_{o}\left[\dot{u}_{i, i}+\Delta \tau_{o} \ddot{u}_{i, i}\right]=\kappa \nabla^{2} T,
\end{aligned}
$$

where $K$ is bulk modulus, $\rho$ is fluid density, $\eta$ is fluid viscosity, $T$ is temperature variable, $T_{o}$ is absolute temperature, $\beta\left(=3 K \alpha_{t}\right)$ is thermal constant, $\alpha_{t}$ is coefficient of linear expansion, $\mathbf{u}$ is displacement vector, $t_{i j}$ and $e_{i j}$ are stress and strain tensors, $\delta_{i j}$ is Kronecker delta, $\tau_{0}$ and $\tau_{1}$ are relaxation times, $\kappa$ is coefficient of thermal conductivity, $C^{*}$ is specific heat at constant strain and the symbol $\Delta$ in (3) makes these fundamental equations possible for the two different theories of the generalized thermoelasticity.

For the LS (Lord-Shulman) theory $\tau_{1}=0, \Delta=1$ and for GL (Green-Lindsay) theory $\tau_{1}>0$ and $\Delta=0$. The thermal relaxation times $\tau_{o}$ and $\tau_{1}$ satisfy the inequality $\tau_{1} \geq \tau_{o} \geq 0$ for the GL theory only.

\section{Solution of the problem}

To solve the basic equations, we decompose the displacement vector as

$$
\mathbf{u}=\nabla \phi+\nabla \times \psi, \quad \nabla \cdot \psi=0,
$$

Using (4), (2) reduces to

$$
\begin{aligned}
v_{1}^{2} \nabla^{2} \phi & =\ddot{\phi}+\bar{\beta}\left(T+\tau_{1} \dot{T}\right), \\
v_{2}^{2} \nabla^{2} \psi & =\ddot{\psi},
\end{aligned}
$$


where

$$
v_{1}^{2}=\left(K+\frac{4}{3} \eta \frac{\partial}{\partial t}\right) / \rho, \quad v_{2}^{2}=\left(\eta \frac{\partial}{\partial t}\right) / \rho, \quad \bar{\beta}=\beta / \rho .
$$

From (5), it is clear that the longitudinal wave is affected due to the thermal disturbances.

From equation (5), we have

$$
T=\left(v_{1}^{2} \nabla^{2} \phi-\ddot{\phi}\right) / \bar{\gamma},
$$

where

$$
\bar{\gamma}=\bar{\beta}\left[1+\tau_{1}(\partial / \partial t)\right],
$$

Eliminating $T$ from (3) and (8), we get

$$
\begin{aligned}
\nabla^{4} \phi- & {\left[\frac{C^{*}}{\bar{\kappa}}\left\{\left(1+\tau_{o} \frac{\partial}{\partial t}\right)+\varepsilon\left(1+\tau_{1} \frac{\partial}{\partial t}\right)\left(1+\Delta \tau_{o} \frac{\partial}{\partial t}\right)\right\}+\frac{1}{v_{1}} \frac{\partial}{\partial t}\right] \frac{\partial}{\partial t} \nabla^{2} \phi } \\
& +\frac{C^{*}}{\bar{\kappa}} \frac{1}{v_{1}^{2}}\left(1+\tau_{o} \frac{\partial}{\partial t}\right) \frac{\partial^{3} \phi}{\partial t^{3}}=0
\end{aligned}
$$

where

$$
\varepsilon=\bar{\beta}^{2} T_{o} / v_{1}^{2} C^{*}, \quad \bar{\kappa}=\kappa / \rho .
$$

We assume the solution of (10) in the form

$$
\phi=f(z) \exp [i k(c t-x)], \quad\left(c>v_{1}\right)
$$

where $k$ is the wave number.

With the help of (12), (10) reduces to

$$
\frac{\mathrm{d}^{4} f(z)}{\mathrm{d} z^{4}}+A \frac{\mathrm{d}^{2} f(z)}{\mathrm{d} z^{2}}+B f(z)=0,
$$

where

$$
\begin{gathered}
A=k^{2}\left(\frac{c^{2}}{v_{1}^{2}}-2\right)-k c\left(C^{*} / \bar{\kappa}\right)\left[\left(i-\tau_{o} k c\right)+\varepsilon_{1}\left(i-\tau_{1} k c\right)\left(1+i k c \tau_{o} \Delta\right)\right], \\
B=k^{4}\left(1-\frac{c^{2}}{v_{1}^{2}}\right)+c k^{3}\left(C^{*} / \bar{k}\right)\left[\left(i-\tau_{o} k c\right)+\varepsilon\left(i-\tau_{1} k c\right)\left(1+i k c \tau_{o} \Delta\right) .\right. \\
\left.\quad-\frac{c^{2}}{v_{1}^{2}}\left(i-\tau_{o} k c\right)\right] .
\end{gathered}
$$

and phase velocity $c$ is equal to $v / \sin I$, where $v$ is the velocity of incident wave and $I$ denotes the angle of incidence.

The solution of (13) is of the form

$$
\begin{gathered}
f(z)=A_{1} \exp \left(i k m_{1} z\right)+A_{2} \exp \left(-i k m_{1} z\right)+A_{3} \exp \left(i k m_{2} z\right) \\
+A_{4} \exp \left(-i k m_{2} z\right)
\end{gathered}
$$


where

$$
\begin{aligned}
& m_{1}^{2}=\left[\left\{\left(A^{2}-4 B\right)^{1 / 2}+A\right\} / 2 k^{2}\right], \\
& m_{2}^{2}=\left[\left\{-\left(A^{2}-4 B\right)^{1 / 2}+A\right\} / 2 k^{2}\right],
\end{aligned}
$$

correspond to the longitudinal wave and thermal wave respectively and $A_{1}, A_{2}, A_{3}, A_{4}$ are arbitrary constants.

Equation (6) can be written as

$$
v_{2}^{2} \nabla^{2} \psi=\ddot{\psi}
$$

where

$$
\psi=(-\vec{\psi})_{y}
$$

We assume the solution of (19) in the form

$$
\psi=g(z) \exp [i k(c t-x)], \quad\left(c>v_{2}\right) .
$$

From (19) and (20), the solution $\psi$ can be written as

$$
\psi=\left[A_{5} \exp \left(i k m_{3} z\right)+A_{6} \exp \left(-i k m_{3} z\right)\right] \exp [i k(c t-x)],
$$

where

$$
m_{3}^{2}=\left(c^{2} / v_{2}^{2}-1\right),
$$

corresponds to transverse wave and A5, A6 are arbitrary constants.

The displacement components of vector $\mathbf{u}$ in $x-z$ plane are

$$
u_{1}=\frac{\partial \phi}{\partial x}+\frac{\partial \psi}{\partial z}, \quad u_{3}=\frac{\partial \phi}{\partial z}-\frac{\partial \psi}{\partial x},
$$

\section{Reflection}

We consider the propagation of plane waves in the $x-z$ plane which makes an angle $I$ with the normal to the boundary. For an incident longitudinal wave, $c=m_{1} \operatorname{cosec} I$ and for an incident transverse wave, $c=m_{3} \operatorname{cosec} I$. For incident longitudinal wave or transverse wave, we get three reflected waves in thermally conducting viscous liquid. The complete geometry showing these reflected has been given in figure 1.

The appropriate potentials are

$$
\begin{aligned}
\phi= & B_{o} \exp \left\{i k\left(c t-x+m_{1} z\right)\right\}+B_{1} \exp \left\{i k\left(c t-x-m_{1} z\right)\right\} \\
& +B_{2} \exp \left\{i k\left(c t-x-m_{2} z\right)\right\}, \\
T= & \left(1 / \bar{\gamma}_{o}\right)\left[a_{1} B_{o} \exp \left\{i k\left(c t-x+m_{1} z\right)\right\}+a_{1} B_{1} \exp \left\{i k\left(c t-x-m_{1} z\right)\right\}\right. \\
& \left.+a_{2} B_{2} \exp \left\{i k\left(c t-x-m_{2} z\right)\right\}\right], \\
\psi= & B_{o} \exp \left\{i k\left(c t-x+m_{3} z\right)\right\}+B_{3} \exp \left\{i k\left(c t-x-m_{3} z\right)\right\},
\end{aligned}
$$


where $B_{i}(i=0,1,2,3)$ are arbitrary constants, and

$$
\begin{aligned}
a_{1,2} & =k^{2}\left\{\left(c^{2}-v_{1}^{2}\right)+m_{1,2}^{2} v_{1}^{2}\right\}, \\
\bar{\gamma}_{o} & =\bar{\beta}\left(1+i \omega \tau_{1}\right),
\end{aligned}
$$

Boundary conditions: For two-dimensional motion in $x-z$ plane, the appropriate boundary conditions at free surface $z=0$, are as

$$
t_{z z}=0, \quad t_{z x}=0, \quad \partial T / \partial z=0,
$$

Making use of the potentials given by equations (24) to (26) in boundary conditions (29), after using the equations (1) and (23), we get a system of three non-homogeneous equations as

$$
\sum_{j=1}^{3} C_{i j} Z_{j}=d_{j}, \quad(i=1,2,3)
$$

where

$$
\begin{aligned}
& C_{11}=-(K+4 \eta i \omega / 3) m_{1}^{2}-(K-2 \eta i \omega / 3)-\rho a_{1} / k^{2}, \\
& C_{12}=-(K+4 \eta i \omega / 3) m_{2}^{2}-(K-2 \eta i \omega / 3)-\rho a_{2} / k^{2}, \quad C_{13}=2 \eta i \omega m_{3}, \\
& C_{21}=-2 m_{1}, \quad C_{22}=-2 m_{2}, \quad C_{23}=\left(1-m_{3}^{2}\right) \\
& C_{31}=m_{1} a_{1}, \quad C_{32}=m_{2} a_{2}, \quad C_{33}=0,
\end{aligned}
$$

and

For incident longitudinal wave

$$
d_{1}=-C_{11}, \quad d_{2}=C_{21}, \quad d_{3}=C_{31},
$$

For incident transverse wave

$$
d_{1}=C_{13}, \quad d_{2}=-C_{23}, \quad d_{3}=C_{33},
$$

and $\left(Z_{j}\right)$ are the amplitude ratios for various reflected waves.

\section{Numerical analysis}

To explain the analytical procedure presented earlier, we now consider a numerical example. The results depict the variation of the angle of incidence with the modulus of the amplitude ratios.

The physical constants are (Fehler 1982)

$$
\begin{aligned}
\rho & =1.01 \mathrm{gm} / \mathrm{cm}^{3}, \quad K=0.0119 \times 10^{11} \mathrm{dyne} / \mathrm{cm}^{2}, \quad \eta=0.0014 \mathrm{gm} / \mathrm{cm} \mathrm{s}, \\
K^{*} & =0.48 \mathrm{cal} / \mathrm{cm} \mathrm{s}^{\circ} \mathrm{C}, \quad C^{*}=0.206 \mathrm{cal} / \mathrm{gm}^{\circ} \mathrm{C}, T_{o}=20^{\circ} \mathrm{C}, \\
\omega & =5 s^{-1}, \quad \varepsilon=0.053,
\end{aligned}
$$

Nayfeh \& Nasser (1971) took $\tau_{o}=3 K^{*} / \rho C^{*} \alpha^{2}$. The relaxation time $\tau_{1}$ is considered to be of same order as that of $\tau_{o}$. The system of equations (30) is solved for amplitude ratios by using a computer program of the Gauss elimination method. The amplitude ratios for reflected longitudinal wave, reflected thermal wave and reflected transverse wave are computed for angle of incidence varying from $0^{\circ}$ to $90^{\circ}$. 

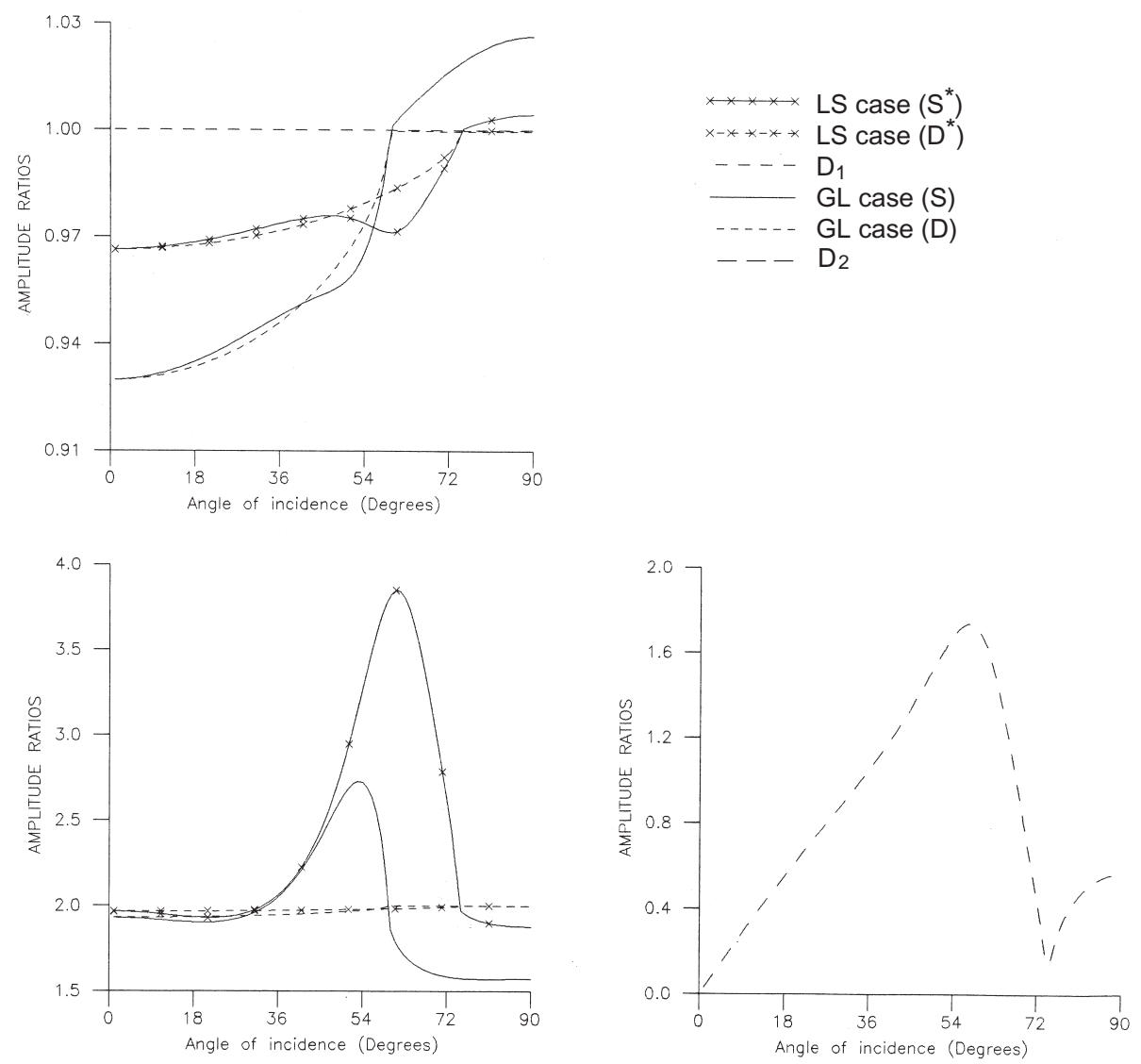

Figure 2. Variations of the amplitude ratios for incident longitudinal wave.

\subsection{Incident longitudinal wave}

The amplitude ratios for reflected longitudinal wave for Lord and Shulman (LS) case (Lord \& Shulman 1967) and Green and Lindsay (GL) case (Green \& Lindsay 1972) are shown by solid curves $S^{*}$ and $S$ in figure 2(a). These curves reduce to dashed curves $D^{*}$ and $D$, if we put $\eta=0$. If we neglect thermal effects, the curves $D^{*}$ and $D$ reduce to curve $D_{1}$.

The amplitude ratios for reflected thermal wave first increase to their maximum values and then decrease sharply. The variations for LS and GL cases are shown by curves $S^{*}$ and $S$ in figure 2(b). These curves reduce to curves $D^{*}$ and $D$, if we neglect viscous effect. This reflected wave would disappear, if we neglect thermal effect also.

The amplitude ratios for reflected transverse wave are shown by curve $D_{2}$ in figure 2(c). It increases to its maximum value and then oscillates. This reflected wave disappears, if we neglect viscous effect.

\subsection{Incident transverse wave}

The variations of the amplitude ratios for reflected longitudinal, thermal and transverse waves are shown in figure 3 . The amplitude ratios for reflected transverse wave first decrease to its minimum value and then oscillate. The variations of amplitude ratios for reflected longitudinal 


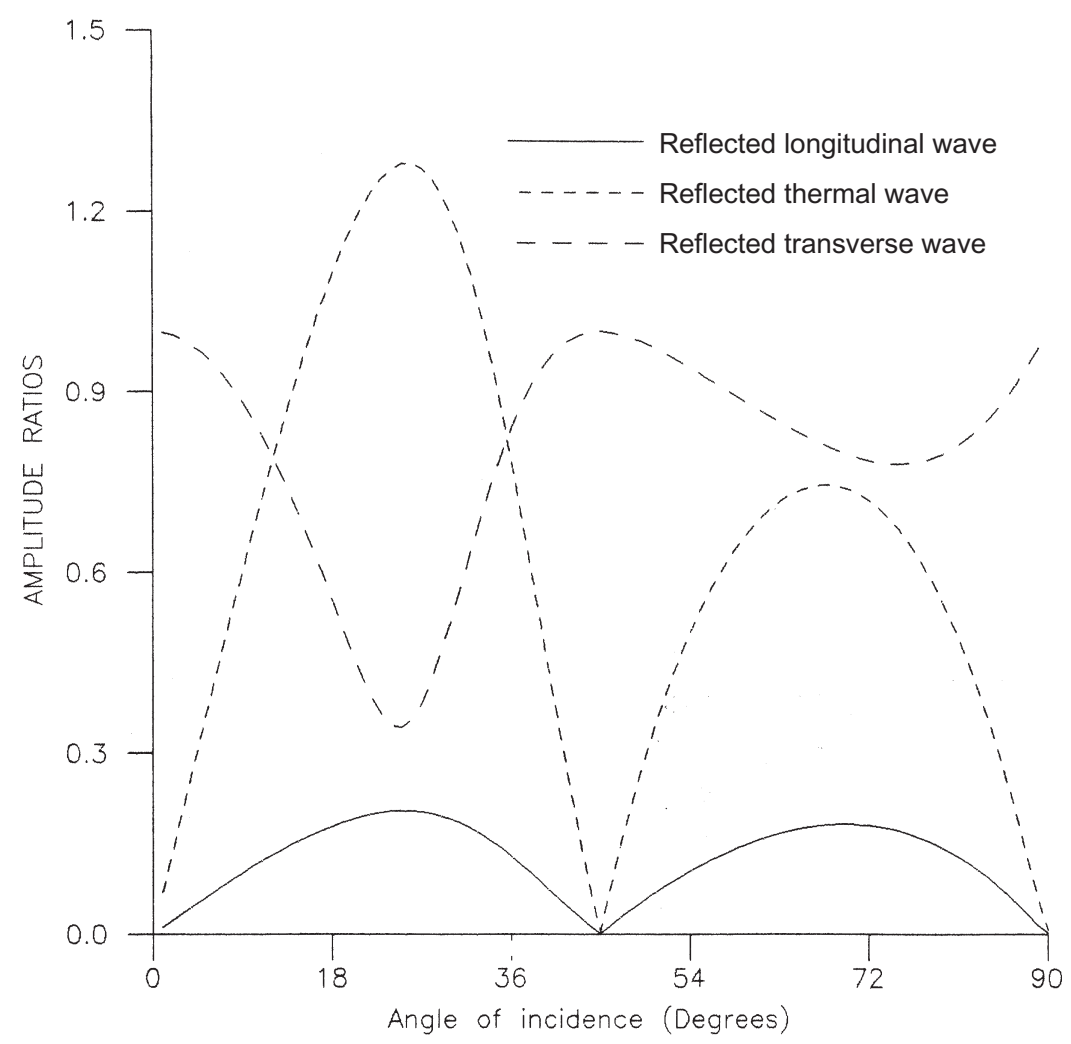

Figure 3. Variations of the amplitude ratios for incident transverse wave.

wave and reflected thermal wave are similar. They first increase to their respective maxima and then oscillate. The variation of the amplitude ratios for reflected longitudinal wave is shown after multiplying its original value by 10 .

From $\S 5.1$, we conclude that the reflected longitudinal and thermal waves are affected by second thermal relaxation in time and viscosity of the liquid. The reflected transverse wave will not exist if we put viscosity of liquid equal to zero. The analysis shown in this section is not possible in a non-viscous liquid.

\section{References}

Achenbach J D 1973 Wave propagation in elastic solids (Amsterdam: North-Holland, Elsevier)

Bath M 1968 Mathematical aspects of seismology (Amsterdam: Elsevier)

Bullen K E 1963 An introduction to the theory of seismology. (London: Cambridge University Press)

Chadwick P, Sneddon I N 1958 Plane waves in an elastic solid conducting heat. J. Mech. Phys. Solids 6: 223-230

Deresiewicz H 1960 Effect of boundaries on waves in a thermoelastic solid: Reflexion of plane waves from plane boundary. J. Mech. Phys. Solids 8: 164-172

Ewing W M, Jardetzky W S, Press F 1957 Elastic waves in layered media (New York, Toronto, London : McGraw Hill)

Fehler M 1982 Interaction of seismic waves with viscous liquid layer. Bull. Seismic Soc. Am. 72: 55-72 
Green A E, Lindsay K A 1972 Thermoelasticity. J. Elasticity 2: 1-7

Kalski H 1963 Stress waves in solids (New York: Dover)

Knot C G 1899 Reflection and refraction of elastic waves with seismological application. Philos. Mag. 5: 64-97

Lockett F J 1958 Effect of the thermal properties of a solid on the velocity of Rayleigh waves. J. Mech. Phys. Solids. 7: 71-75

Lord H, Shulman Y 1967 A generalized dynamical theory of thermoelasticity. J. Mech. Phys. Solids. 15: $299-309$

Miklowitz J 1966 Elastic wave propagation (Applied Mechanics Survey) (Spartan Books)

Nayfeh A, Nasser S N 1971 Thermoelastic waves in solids with thermal relaxation. Acta Mech. 12: 53-69

Singh B 2000 Reflection of plane sound wave from a micropolar generalized thermoelastic solid halfspace. J. Sound Vibr. 235: 685-696

Sinha A N, Sinha S B 1974 Reflection of thermoelastic waves at a solid half-space with thermal relaxation. J. Phys. Earth 22: 237-244 